Qualitative-other

\section{People with coexisting diabetes, chronic kidney disease and hypertension report the psychosocial effects of being prescribed multiple medications}

10.1136/eb-2014-101829

\section{Alan J Zillich}

Department of Pharmacy Practice, Purdue University College of Pharmacy, Indianapolis, Indiana, USA

Correspondence to: Dr Alan J Zillich, Department of Pharmacy Practice, Purdue Pharmacy Programs, Purdue University College of Pharmacy, 5th/ 3rd Bank Faculty Office Building, 640 Eskenazi Avenue, Indianapolis, IN 46202, USA; azillich@purdue.edu

Commentary on: Williams A, Manias E. Exploring motivation and confidence in taking prescribed medicines in coexisting disease: a qualitative study. J Clin Nurs 2014;23:471-81.

\section{Implications for practice and research}

- Patients' health and medication beliefs contribute to intentional medication non-adherence.

- Interventions to improve intentional medication non-adherence must be patient-centred. Strategies such as team-based care and motivational interviewing should be considered.

\section{Context}

Adherence to prescribed medication is estimated to occur in only 50\% of patients. As the numbers of comorbid conditions and medications increase, the risk of medication non-adherence also increases. Interventions to improve medication adherence are often complex, multifactorial and individualised. ${ }^{1}$ Rooted in this individualised approach is a need to understand the behavioural motivations and challenges that influence patient adherence to medicines. One proposed intervention strategy utilises motivational interviewing (MI) to explore and resolve resistance to medication adherence. ${ }^{2}$ In this study, Williams and Manias examined patients' responses during an MI intervention designed to improve medication adherence.

\section{Methods}

The purpose of the study was to explore the motivation and confidence of patients with coexisting diabetes, hypertension and chronic kidney disease to take their medication as prescribed. As part of a randomised controlled trial investigating a programme to improve adherence, patients in the intervention group $(n=39)$ received a series of MI phone calls every 2 weeks during a 12-week period. During the calls, an intervention nurse, trained in MI, took handwritten notes to capture the patients' responses. The handwritten notes for all patients' calls $(n=164)$ were transcribed and used for the qualitative analyses. The thematic analyses incorporated the
Health Belief Model. Transcripts were independently reviewed by two investigators until saturation of themes and subthemes occurred.

\section{Findings}

Patients were an average of 68 years old and taking an average 7.6 medications daily. Transcribed data of 749 free nodes were organised into themes that mapped to motivational categories of the modified Health Belief Model, including: the importance of health, the perceived seriousness of disease, psychosocial and interpersonal aspects, perceived benefits, and barriers to action. Patients' motivation to take medication as prescribed was hindered by their chronic and sometimes asymptomatic disease, the complexity of their medication regimen, and the interpersonal relationship with healthcare providers.

\section{Commentary}

Often, patients' diseases and disease beliefs can hinder medication adherence, particularly when a disease is largely asymptomatic. In a crosssectional study of 24017 patients, 8\% of respondents took less medication than prescribed because they felt better, while 34.3\% took less medication due to a variety of health and medication beliefs. ${ }^{3}$ This type of medication non-adherence is often described as intentional nonadherence, reflecting active, rational decision-making on the part of the patient to not take their medication as prescribed.

Herein lays a challenge for healthcare providers to improve medication adherence by reducing intentional non-adherence. Interventions for intentional non-adherence must compliment the health beliefs of the patient with empathy, empowerment and knowledge. Interpersonal communication and relationship building between healthcare providers and patients must occur.

Williams and Manias described patient motivation in relation to medication adherence as being reliant on a partnership between the patient and healthcare providers. Lack of sensitive communication and trust between patients and healthcare providers led to non-adherence. These findings support the use of strategies such as MI and interprofessional, team-based care to improve patient adherence. ${ }^{4}$ Indeed, systematic reviews of practitioners such as nurses and pharmacists have demonstrated that when these clinicians are part of the healthcare team, patient adherence and outcomes improve. ${ }^{5}{ }^{6}$ Our healthcare systems must strive to collaborate across healthcare disciplines to address the complex problems associated with non-adherence to medication.

Competing interests None.

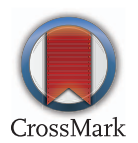

\section{References}

1. Haynes R, Ackloo E, Sahota N, et al. Interventions for enhancing medication adherence. Cochrane Database Syst Rev 2008;2:CD000011.

2. Miller W, Rollnick S. Motivational interviewing: preparing people for change. New York, NY: Guilford Press, 2002.

3. Gadkari AS, McHorney CA. Unintentional non-adherence to chronic prescription medications: how unintentional is it really? BMC Health Serv Res 2012;12:98.

4. Mitchell P, Wynia M, Golden R, et al. Core principles \& values of effective team-based health care. Washington, DC: Institute of Medicine, 2012.

5. Van Camp YP, Van Rompaey B, Elseviers MM. Nurse-led interventions to enhance adherence to chronic medication: systematic review and meta-analysis of randomised controlled trials. Eur J Clin Pharmacol 2013;69:761-70.

6. Chisholm-Burns MA, Lee JK, Spivey CA, et al. US Pharmacists' effect as team members on patient care: systematic review and meta-analyses. Med Care 2010;48:923-33. 